PROCEEDINGS OF THE

AMERICAN MATHEMATICAL SOCIETY

Volume 136, Number 6, June 2008, Pages 1911-1918

S 0002-9939(08)09165-X

Article electronically published on February 13, 2008

\title{
DIFFERENTIAL CENTRAL SIMPLE ALGEBRAS AND PICARD-VESSIOT REPRESENTATIONS
}

\author{
LOURDES JUAN AND ANDY R. MAGID
}

(Communicated by Martin Lorenz)

\begin{abstract}
A differential central simple algebra, and in particular a differential matrix algebra, over a differential field $K$ with constants $C$ can be trivialized by a Picard-Vessiot (differential Galois) extension $E$. In the matrix algebra case, there is a correspondence between $K$-algebras trivialized by $E$ and representations of the Galois group of $E$ over $K$ in $\mathrm{PGL}_{n}(C)$, which can be interpreted as cocyles equivalent up to coboundaries.
\end{abstract}

\section{INTRODUCTION}

Let $K$ be a differential field with algebraically closed characteristic zero field of constants $C$ and derivation $D=D_{K}$. By a differential central simple algebra (DCSA) over $K$ we mean a pair $(A, \mathcal{D})$ where $A$ is a central simple $K$-algebra and $\mathcal{D}$ is a derivation of $A$ extending the derivation $D$ of its center $K$. Given two such pairs $\left(A_{1}, \mathcal{D}_{1}\right)$ and $\left(A_{2}, \mathcal{D}_{2}\right)$, we can form the differential algebra $\left(A_{1} \otimes A_{2}, \mathcal{D}_{1} \otimes \mathcal{D}_{2}\right)$, where the derivation $\mathcal{D}_{1} \otimes \mathcal{D}_{2}$ is given by $\mathcal{D}_{1} \otimes 1+1 \otimes \mathcal{D}_{2}$. Morphisms of DCSAs over $K$ are $K$-algebra homomorphisms preserving derivations. We denote the isomorphism class of $(A, \mathcal{D})$ by $[A, \mathcal{D}]$. The above tensor product defines a monoid operation on the isomorphism classes (the identity being the class $\left[K, D_{K}\right]$ ), and hence we can form the corresponding universal group $K_{0}^{\text {diff }} \mathcal{A} z(K)$. (A central simple algebra (CSA) over a field is a special case of Azumaya, or central separable, algebras over rings; $\mathcal{A} z$ is an abbreviation for Azumaya.) The corresponding object for $K$ when derivations are not considered is the familiar $K_{0} \mathcal{A} z(K)$ as defined by Bass in 1, Chapter III]. There is an obvious group homomorphism $K_{0}^{\text {diff }} \mathcal{A} z(K) \rightarrow K_{0} \mathcal{A} z(K)$ given by $[A, \mathcal{D}] \mapsto[A]$. This group homomorphism is surjective: any derivation, such as $D_{K}$, of the center of any central simple $K$-algebra $A$ can be extended to a derivation of $A$. (For Azumaya algebras in general, this is Hochschild's Theorem; see [5].) These extensions can be done in many ways (we recall, for later use, that any two differ by a derivation trivial on the center, and hence inner 1 , 1.6 , p. 86]). For the matrix algebras $M_{n}(K)$, we have the extension which is given by $D_{K}$ on the matrix coordinates, and we denote the corresponding differential algebra by $\left(M_{n}(K),()^{\prime}\right)$. We note that under the usual isomorphism from $M_{p}(K) \otimes M_{q}(K) \rightarrow M_{p q}(K)$ we have $\left(M_{p}(K),(\quad)^{\prime}\right) \otimes\left(M_{q}(K),(\quad)^{\prime}\right)$ isomorphic to $\left(M_{p q}(K),(\quad)^{\prime}\right)$.

Received by the editors September 15, 2006.

2000 Mathematics Subject Classification. Primary 12H05; Secondary 16H05.

(C)2008 American Mathematical Society 
The group homomorphism $K_{0} \mathcal{A} z(K) \rightarrow \mathcal{B} r(K)$ to the Brauer group [1, 6.2, p. 113] of $K$ is the surjection with kernel the subgroup $K_{0} \mathcal{M} a t(K)$ generated by the matrix algebras. We consider the corresponding subgroup $M^{1}$ of $K_{0}^{\text {diff }} \mathcal{M}$ at $(K)$ generated by the algebras $\left(M_{n}(K),()^{\prime}\right)$ and its quotient $\mathcal{B} r^{\text {diff }}(K)$, which we call the differential Brauer group of $K$. This is of interest even in the case that $K$ is algebraically closed and hence $\mathcal{B} r(K)$ is trivial. Then $\mathcal{B} r^{\text {diff }}(K)$ reduces to $M^{2} / M^{1}$, where the group $M^{2}$ is generated by the DCSAs $\left[M_{n}(K), \mathcal{D}\right]$ whose $K$ algebra component is a matrix algebra and this quotient can be non-trivial.

Even such an algebraically closed $K$ can still have non-trivial differential Galois extensions. For any given DCSA $(A, \mathcal{D})$, there is a finite Galois (hence also uniquely differential Galois) base extension $E$ of $K$ such that with this extension of scalars $A$ becomes a matrix algebra and $(A, \mathcal{D}) \otimes E$ lies in $M^{2}$ in $K_{0}^{\text {diff }} \mathcal{A} z(E)$. We thus concentrate on the group $M^{2} / M^{1}$. Once this group is analyzed we return to the general situation in our final section. In this context, the algebras $\left(M_{n}(K),(\quad)^{\prime}\right)$ play the role of matrix algebras in the non-differential theory, and hence we will refer to them as the trivial DCSAs.

We show that any given differential matrix algebra is trivialized by a PicardVessiot extension (PVE), and that therefore such algebras are given by representations of the differential Galois group of the extension in the automorphism group of the trivial algebra over the extension, which we show amounts to a representation into $\mathrm{PGL}_{n}(C)$. These are interpreted as cocycles, and we show how the cocyle is determined up to coboundary.

We retain throughout the paper the terminology, notation and conventions of this introduction. We are grateful to the referee of an earlier version of this paper for helpful suggestions.

\section{Differential matrix Algebras}

We begin with an analysis of the structure of a DCSA whose underlying algebra is a matrix algebra.

Definition 1. Let $P$ be an $n \times n$ matrix over $K$. Then $\mathcal{D}_{P}$ denotes the derivation of $M_{n}(K)$ given by

$$
\mathcal{D}_{P}(X)=(X)^{\prime}+P X-X P .
$$

Note that $\mathcal{D}_{P}=\mathcal{D}_{Q}$ implies that $P-Q$ is central, and hence scalar, so $P=$ $Q+a I_{n}$ for suitable $a \in K$, and conversely. So $D_{P}$ determines $P$ provided that the trace of $P$ is specified (we are especially grateful to the referee for this observation). Thus we generally specify that $P$ is of trace 0 .

We further note that

$$
\left(M_{n}(K), \mathcal{D}_{P}\right) \otimes\left(M_{m}(K), \mathcal{D}_{Q}\right)=\left(M_{n m}(K), \mathcal{D}_{P \otimes I_{m}+I_{n} \otimes Q}\right) .
$$

It is a simple consequence of the fact that central derivations of CSAs are inner to see that all matrix DCSAs have such derivations:

Proposition 1. Let $\left(M_{n}(K), \mathcal{D}\right)$ be a DCSA over $K$. Then there is a matrix $P \in M_{n}(K)$ such that $\mathcal{D}=\mathcal{D}_{P}$.

Proof. $\mathcal{D}-()^{\prime}$ is a central derivation of $M_{n}(K)$, and hence inner. If this inner derivation is given by $P \in M_{n}(K)$, then $\mathcal{D}(X)-(X)^{\prime}=P X-X P$, so that $\mathcal{D}=\mathcal{D}_{P}$. 
From now on, we will denote a matrix DCSA as $\left(M_{n}(K), \mathcal{D}_{P}\right)$, where it is understood that $P$ is of trace zero. Using this structure, we consider when a matrix DCSA is trivial.

Proposition 2. $\left(M_{n}(K), \mathcal{D}_{P}\right)$ is isomorphic to $\left(M_{n}(K), \mathcal{D}_{Q}\right)$ if and only if there is $H \in \mathrm{GL}_{n}(K)$ such that $H^{-1} H^{\prime}+H^{-1} Q H=P$. In particular, $\left(M_{n}(K), \mathcal{D}_{P}\right)$ is isomorphic to $\left(M_{n}(K),()^{\prime}\right)$ if and only if there is $F \in \mathrm{GL}_{n}(K)$ such that $F^{-1}(F)^{\prime}=P$.

Proof. Suppose $T:\left(M_{n}(K), \mathcal{D}_{P}\right) \rightarrow\left(M_{n}(K), \mathcal{D}_{Q}\right)$ is a differential $K$-algebra isomorphism. As a $K$-algebra isomorphism (actually automorphism) of $M_{n}(K), T$ is inner 6, Thm. 2.10, p.16]: there is $H \in \mathrm{GL}_{n}(K)$ such that $T(X)=H X H^{-1}$. Since $H$ is only defined up to a non-zero scalar multiple, we can assume that $\operatorname{det}(H)=1$. Since $T$ is differential, we have $T\left(\mathcal{D}_{P}(X)\right)=\mathcal{D}_{Q}(T(X))$. Expanding and simplifying, this implies that $H^{-1} H^{\prime}+H^{-1} Q H-P$ commutes with $X$, hence is central, so of the form $b I_{n}$. Taking traces, we see that $n b=\operatorname{tr}\left(H^{-1} H^{\prime}\right)$. Since $\operatorname{tr}\left(H^{-1} H^{\prime}\right)$ is the logarithmic derivative of the determinant of $H, b=0$.

Conversely, suppose that there is an $H \in \mathrm{GL}_{n}(K)$ such that $P=H^{-1} H^{\prime}+$ $H^{-1} Q H$. One can check that the inner automorphism $T_{H}: M_{n}(K) \rightarrow M_{n}(K)$ by $T_{H}(X)=H X H^{-1}$ is a differential isomorphism $\left(M_{n}(K), \mathcal{D}_{P}\right) \rightarrow\left(M_{n}(K), \mathcal{D}_{Q}\right)$ as required.

If $E \supseteq K$ is a differential extension field, and $(A, \mathcal{D})$ is a DCSA over $K$, then $\left(A \otimes_{K} E, \mathcal{D} \otimes_{K} D_{E}\right)$, where $D_{E}$ is the derivation of $E$ and $\mathcal{D} \otimes_{K} D_{E}=\mathcal{D} \otimes 1+1 \otimes D_{E}$, is a differential central simple $E$-algebra. In case $A=M_{n}(K)$, we identify $A \otimes_{K} E$ with $M_{n}(E)$ as usual. The proposition gives a criterion to tell whether $\left(M_{n}(K), \mathcal{D}\right)$ becomes trivial after extending scalars to $E$. To make this explicit, we turn to PVEs.

The matrix equation $Q=F^{-1} F^{\prime}$ means that $F$ is a solution of the matrix differential equation $Y^{\prime}=Y Q$, and conversely any solution $F$ of the latter satisfies $Q=F^{-1} F^{\prime}$.

A PVE of $K$ for the matrix differential equation $Y^{\prime}=R Y, R \in M_{n}(K)$, is a no new constant differential field extension $E \supseteq K$ such that the equation has an invertible matrix solution (a matrix $F \in \mathrm{GL}_{n}(E)$ such that $F^{\prime}=R F$ ), and which is minimal over $K$ with this property ( $E$ is differentially generated over $K$ by the entries of $F$ ).

Theorem 1. Let $\left(M_{n}(K), \mathcal{D}\right)$ be a differential matrix algebra over $K$. Then there is a $P V E E \supseteq K$ such that $\left(M_{n}(K), \mathcal{D}\right) \otimes_{K} E$ is trivial.

Proof. We choose trace zero $P \in M_{n}(K)$ so that $\mathcal{D}=\mathcal{D}_{P}$. Then we let $E \supseteq K$ be a PVE for the equation $Y^{\prime}=P^{t} Y$. By definition, there is $G \in \mathrm{GL}_{n}(E)$ such that $G^{\prime}=P^{t} G$. Let $F=G^{t}$. Then $F^{\prime}=F P$, so by Proposition 2 we have that $\left(M_{n}(K), \mathcal{D}\right) \otimes_{K} E$ is trivial.

\section{Automorphisms of matrix Differential Algebras}

We begin by considering a differential automorphism $T$ of $\left(M_{n}(K),(\quad)^{\prime}\right)$ over $K$. As an algebra homomorphism, $T$ is inner, say given by conjugation by the invertible matrix $A$. For conjugation by $A$ to be a differential homomorphism, we must have $\left(A X A^{-1}\right)^{\prime}=A X^{\prime} A^{-1}$ for all $X \in M_{n}(E)$. Expanding the left-hand side of the equation, and pre- and post-multiplying by $A^{-1}$ and $A$, the equation 
becomes $A^{-1} A^{\prime} X-X A^{-1} A^{\prime}=0$, from which it follows that $A^{-1} A^{\prime}$ is central, say equals $a I_{n}$. If $a=0$, then $A$ is in $\mathrm{GL}_{n}(C)$. If not, we enlarge $K$ if necessary by a PVE $L$ adjoining a (non-zero) element $b$ with $b^{\prime}=a b$. Then $\left(b^{-1} A\right)^{\prime}=0$, and an inner automorphism by the matrix of constants $b^{-1} A$ on $M_{n}(L)$ is the same as an inner automorphism by $A$. Thus in any case, $T$ on $M_{n}(K)$ is given by an inner automorphism by a constant matrix. This latter is only determined up to a non-zero scalar multiple, i.e. by an element of $\mathrm{PGL}_{n}(C)$. Conversely, elements $A$ of this group yield differential automorphisms of $\left(M_{n}(K),()^{\prime}\right)$ over $K$, which we will denote by abuse of notation as the inner automorphism $T_{A}$.

Now let $E \supset K$ be a PVE with group $G=G(E / K)$. Suppose that $S$ is a $K$ linear differential algebra automorphism of $\left(M_{n}(E),()^{\prime}\right)$. T preserves the center $E$ of $M_{n}(E)$, and on $E, S$ is given by some element $\sigma$ of $G$. Let $M_{n}(\sigma)$ denote the extension of $\sigma$ to $M_{n}(E)$ by action on coordinates. Then $S M_{n}(\sigma)^{-1}$ is a differential automorphism on $M_{n}(E)$ trivial on $E$, hence by the above given by conjugation by a constant matrix and hence by an element $A$ of $\mathrm{PGL}_{n}(C)$. So $S=T_{A} M_{n}(\sigma)$. Conversely, given any $A \in \mathrm{PGL}_{n}(C)$ and any $\sigma \in G, T_{A} M_{n}(\sigma)$ is a differential algebra automorphism of $\left(M_{n}(K),()^{\prime}\right)$ over $K$. (Note that since $A$ is a constant matrix, in $M_{n}(K), M_{n}(\sigma)$ and $T_{A}$ commute.) We summarize:

Proposition 3. Let $E \supset K$ be a PVE with group $G(E / K)$. The group of differential automorphisms of $\left(M_{n}(E),(\quad)^{\prime}\right)$ over $K$ is isomorphic to $\mathrm{PGL}_{n}(C) \times G(E / K)$. If $A \in \mathrm{PGL}_{n}(C)$ and $\sigma \in G(E / K)$, then the corresponding automorphism is $X \mapsto A \sigma(X) A^{-1}$.

Suppose that $\left(M_{n}(K), \mathcal{D}\right)$ is a matrix differential algebra which becomes trivial in the PVE $E \supseteq K$, so that $\left(M_{n}(K), \mathcal{D}\right) \otimes_{K} E$ is isomorphic to $\left(M_{n}(E),(\quad)^{\prime}\right)$. There is an action of $G=G(E / F)$ on $\left(M_{n}(K), \mathcal{D}\right) \otimes_{K} E$ by $1 \otimes G$, whose fixed ring is $\left(M_{n}(K), \mathcal{D}\right)$, and hence there is an action of $G$ on $\left(M_{n}(E),(\quad)^{\prime}\right)$ whose fixed ring is $\left(M_{n}(K), \mathcal{D}\right)$. We have determined the automorphism group of $\left(M_{n}(E),()^{\prime}\right)$ in Proposition 3 to be $\mathrm{PGL}_{n}(C) \times G(E / K)$, so the above $G$ action is given by a homomorphism to this product group. We now determine that homomorphism.

The algebra $\left(M_{n}(K), \mathcal{D}\right) \otimes_{K} E$ may be described concretely as follows: choose $P \in M_{n}(K)$ such that $\mathcal{D}=\mathcal{D}_{P}$. Then $M_{n}(K) \subseteq M_{n}(E)$ and we can regard $P$ as belonging to the latter so

$$
\left(M_{n}(K), \mathcal{D}\right) \otimes_{K} E=\left(M_{n}(E), \mathcal{D}_{P}\right) .
$$

If $\sigma \in G$ and $X \in M_{n}(E)$, we let $\sigma(X)$ denote the action on coordinates $\left(M_{n}(\sigma)(X)\right)$. Under the above identification, this is the same as the (differential automorphism) action of $1 \otimes \sigma$ on $\left(M_{n}(K), \mathcal{D}\right) \otimes_{K} E$. Note that $\mathcal{D}_{P}(\sigma(X))=$ $\sigma(X)^{\prime}+P \sigma(X)-\sigma(X) P$ and $\sigma\left(\mathcal{D}_{P}(X)\right)=\sigma\left(X^{\prime}\right)+\sigma(P) \sigma(X)-\sigma(X) \sigma(P)$ are equal, since $\sigma$ commutes with derivation on $E$ and $P \in M_{n}(K)$ is fixed by $\sigma$.

We have that $\left(M_{n}(E), \mathcal{D}_{P}\right)$ and $\left(M_{n}(E),()^{\prime}\right)$ are isomorphic by means of an inner isomorphism $T_{F}$ for some $F \in \mathrm{GL}_{n}(E)$ where $F^{-1} F^{\prime}=P$. Thus the above $G$ action on $\left(M_{n}(E), \mathcal{D}_{P}\right)$ can be transported to a $G$ action on $\left(M_{n}(E),()^{\prime}\right)$ via $T_{F}$, so that $\sigma \in G$ acts via $T_{F} M_{n}(\sigma) T_{F}^{-1}$. We denote this action by ${ }^{\sigma} X, X \in M_{n}(E)$, so ${ }^{\sigma} X=F\left(\sigma\left(F^{-1} X F\right)\right) F^{-1}$.

We recall that $F$ satisfies the differential equation $F^{\prime}=F P$. This implies that, for $\sigma \in G, \sigma(F)^{\prime}=\sigma(F) P$. It follows that $\left(\sigma(F) F^{-1}\right)^{\prime}=0$ so that $\sigma(F)={ }_{\sigma} D F$ with ${ }_{\sigma} D \in \mathrm{GL}_{n}(C)$. Thus ${ }^{\sigma} X=F\left(\sigma\left(F^{-1} X F\right)\right) F^{-1}$ equals ${ }_{\sigma} D^{-1} \sigma(X)_{\sigma} D$. 
Thus the differential algebra automorphism $X \mapsto{ }^{\sigma} X$ of $\left(M_{n}(E),(\quad)^{\prime}\right)$ is given by the composition $\operatorname{Inn}\left({ }_{\sigma} D^{-1}\right) \circ M_{n}(\sigma)$ of inner automorphisms by ${ }_{\sigma} D^{-1}$ following the action of $\sigma$ on coordinates, and this describes the desired homomorphism from $G$ to the automorphisms of $\left(M_{n}(E),(\quad)^{\prime}\right)$.

We summarize the above calculations in the following proposition:

Proposition 4. Let $E \supseteq K$ be a $P V E$ with group $G=G(E / K)$ and let $P \in M_{n}(K)$ and $F \in \mathrm{GL}_{n}(E)$ be such that $F^{\prime}=F P$. For $\sigma \in G$ let ${ }_{\sigma} D=\sigma(F) F^{-1}$ in $\mathrm{GL}_{n}(C)$. Then $\sigma \rightarrow\left(\operatorname{Inn}_{\sigma} D^{-1}, \sigma\right)$ represents $G$ as automorphisms so that the differential isomorphism $T_{F}:\left(M_{n}(E), \mathcal{D}_{P}\right) \rightarrow\left(M_{n}(E),(\quad)^{\prime}\right), T_{F}(X)=F X F^{-1}$, is $G$-equivariant, when $G$ acts on the codomain via the above representation and on the domain via the action on coordinates.

\section{Cocycles}

In the direct product description of the differential automorphisms of $\left(M_{n}(E)\right.$, ()$\left.^{\prime}\right)$ which lie over the automorphims $G(E / K)$ of the center, the representation of the latter from Proposition 4 is given as $\sigma \mapsto\left(T_{\sigma} D^{-1}, \sigma\right)$. More generally, a homomorphism $G \rightarrow \mathrm{PGL}_{n}(C) \times G(E / K)$ such that the second coordinate of the image of $\sigma$ is $\sigma$ is given by $\sigma \mapsto(\Phi(\sigma), \sigma)$ where $\Phi: G \rightarrow \mathrm{PGL}_{n}(C)$ is a one-cocyle (here a homomorphism, since $G$ acts trivially), and conversely. In the case of the algebra $\left(M_{n}(E), \mathcal{D}_{P}\right)$ of Proposition 4 this cocycle is $\Phi: G \rightarrow \operatorname{PGL}_{n}(C)$ by $\sigma \mapsto$ $T_{\sigma D^{-1}}$. We note that this is algebraic: recall that ${ }_{\sigma} D$ is defined by $\sigma(F)={ }_{\sigma} D F$, and that $F^{\prime}=F P$. Taking transposes, we have that $\sigma\left(F^{t}\right)=F^{t}{ }_{\sigma} D^{t}$ and that $\left(F^{t}\right)^{\prime}=P^{t} F^{t}$. So we recognize $\sigma \mapsto{ }_{\sigma} D^{t}$ as the representation of $G \rightarrow \mathrm{GL}_{n}(C)$ associated with the PVE of $K$ generated by the entries of $F^{t}$ [7, (2), p. 19] and hence algebraic.

We consider the effect of an isomorphism $\left(M_{n}(K), \mathcal{D}_{P}\right) \rightarrow\left(M_{n}(K), \mathcal{D}_{Q}\right)$ on the above association. By Proposition 2 such an isomorphism is given by conjugation by a matrix $H$ such that $P=H^{-1} H^{\prime}+H^{-1} Q H$. This implies that $\left(F H^{-1}\right)^{-1}\left(F H^{-1}\right)^{\prime}=Q$, so the association $P \rightarrow F$ becomes $Q \rightarrow F H^{-1}$.

The inclusion of $\mathrm{PGL}_{n}(C)$ in $\mathrm{PGL}_{n}(E)$ is $G$-equivariant, so we have an associated map

$$
\operatorname{Hom}\left(G, \mathrm{PGL}_{n}(C)\right)=Z^{1}\left(G, \mathrm{PGL}_{n}(C)\right) \rightarrow Z^{1}\left(G, \mathrm{PGL}_{n}(E)\right)
$$

of cocycle sets. (Cocyles in $Z^{1}\left(G, \mathrm{PGL}_{n}(E)\right)$ are rational functions $\Psi$ such that $\Psi(\sigma \tau)=\Psi(\sigma) \sigma(\Psi(\tau))$.) In the case of Proposition 4, since ${ }_{\sigma} D^{-1}=F \sigma\left(F^{-1}\right)$, we have that the cocycle $\Phi$ is actually a coboundary given by $F^{-1}$ : we define $B^{1}\left(G, \mathrm{PGL}_{n}(E)\right)$ to be the functions $G \rightarrow \mathrm{PGL}_{n}(E)$ given by $\sigma \mapsto J \sigma(J)^{-1}$ for some $J \in \mathrm{PGL}_{n}(E)$. (Note that this is the correct side for the $G$ action to agree with our definition of cocycle.) Under the isomorphism $\left(M_{n}(K), \mathcal{D}_{P}\right) \rightarrow\left(M_{n}(K), \mathcal{D}_{Q}\right)$ given by conjugation by $H$, the corresponding cocycle is the coboundary given by $\left(F H^{-1}\right)^{-1}$, or $\sigma \mapsto F H^{-1} \sigma\left(\left(F H^{-1}\right)^{-1}\right)$. When $H \in \mathrm{GL}_{n}(K)$, so $\sigma(H)=H$, this shows that the coboundaries given by $F^{-1}$ and $\left(F H^{-1}\right)^{-1}$ are equal.

If the matrix $M$ in $\mathrm{GL}_{n}(E)$ represents $J \in \mathrm{PGL}_{n}(E)$, then so does $a M$ for any non-zero $a \in E$, and $a M \sigma(a M)^{-1}=a \sigma(a)^{-1} M \sigma(M)^{-1}$.

This observation will be relevant when we prove the converse of the above cocyle construction, which is the main result of this section.

Theorem 2. Let $E \supseteq K$ be a PVE with differential Galois group $G$, and let $\mathrm{PGL}_{n}(C)$ be represented as the group of inner differential automorphisms of 
$\left(M_{n}(E),()^{\prime}\right)$. Then there is a one-to-one correspondence between $K$-isomorphism classes of matrix differential $K$-algebras trivialized by $E$ and homomorphisms $G \rightarrow$ $\mathrm{PGL}_{n}(C)$ which, as cocycles in $Z^{1}\left(G, \mathrm{PGL}_{n}(E)\right)$, are coboundaries.

In particular, if $\left(M_{n}(K), \mathcal{D}\right)$ is such a $K$-algebra, with $\mathcal{D}=\mathcal{D}_{P}$ and $F \in$ $\mathrm{GL}_{n}(E)$ such that $P=F^{-1} F^{\prime}$, the corresponding cocycle is $X \mapsto{ }^{\sigma} X$ where ${ }^{\sigma} X={ }_{\sigma} D^{-1} \sigma(X)_{\sigma} D$ and ${ }_{\sigma} D \in \mathrm{GL}_{n}(C)$ is $\sigma(F) F^{-1}$; this cocycle is the coboundary associated to $F^{-1}$.

Also, if $\Lambda: G \rightarrow \mathrm{PGL}_{n}(C)$ is a cocyle which is the coboundary associated to a matrix $F^{-1}$, for $\sigma \in G$, let $\rho(\sigma)=\Lambda(\sigma) M_{n}(\sigma)$ be the corresponding differential automorphism of $\left(M_{n}(E),()^{\prime}\right)$ reducing to $\sigma$ on the center. Then $M_{n}(E)^{\rho(G)}=$ $F M_{n}(K) F^{-1}$. Let $Q=F^{-1} F^{\prime}$. Then $M_{n}(K)$ is $\mathcal{D}_{Q}$ stable, and

$$
T_{F^{-1}}:\left(M_{n}(K), \mathcal{D}_{Q}\right) \rightarrow\left(M_{n}(E)^{\rho(G)},(\quad)^{\prime}\right)
$$

is an isomorphism, $\left(M_{n}(K), \mathcal{D}_{Q}\right)$ is trivialized by $E$, and $\Lambda$ is the associated cocycle.

Proof. The main assertion of the theorem follows from the two particular assertions. The first one is established in Proposition 4 . For the second, we introduce the following notation: let $\Lambda(\sigma)$ be given by inner automorphisms by $C(\sigma) \in \mathrm{GL}_{n}(C)$. Then $\Lambda$ being the coboundary associated to $F^{-1}$ means that $F^{-1} \sigma(F)=d_{\sigma} C(\sigma)$ for some $d_{\sigma} \in E$. We usually write this as $\sigma(F)=d_{\sigma} F C(\sigma)$. Since

$$
\rho(\sigma)(X)=\Lambda(\sigma)(\sigma(X))=C(\sigma) \sigma(X) C(\sigma)^{-1}
$$

we have

$$
\begin{gathered}
F C(\sigma) \sigma(X) C(\sigma)^{-1} F^{-1}=\sigma\left(F X F^{-1}\right), \text { or } \\
T_{F}(\rho(\sigma)(X))=\sigma\left(T_{F}(X)\right) .
\end{gathered}
$$

Thus $X$ is $\rho(G)$-invariant if and only if $T_{F}(X)$ is $G$-invariant (under the coordinate action). Since the $G$-invariants of $M_{n}(E)$ under the coordinate action are $M_{n}(K)$, we have that $F^{-1} M_{n}(K) F=M_{n}(E)^{\rho(G)}$. Let $S=F^{-1}$ and consider the isomorphism $T_{S}: M_{n}(K) \rightarrow M_{n}(E)^{\rho(G)}$. Let $\mathcal{D}_{0}$ denote $(\quad)^{\prime}$ on $M_{n}(E)$ restricted to $M_{n}(E)^{\rho(G)}$. By transport of structure, we define a derivation $\mathcal{D}$ on $M_{n}(K)$ by $\mathcal{D}(X)=T_{S} \circ \mathcal{D}_{0} \circ T_{S}{ }^{-1}$. Then $\mathcal{D}(X)=F^{-1}\left(F X F^{-1}\right)^{\prime} F$. Thus $\mathcal{D}=\mathcal{D}_{Q}$ where $Q=F^{-1} F^{\prime}$. (We note here that $Q$ is not necessarily in $M_{n}(K)$, although we must have $Q=a I_{n}+P$ for some $P \in M_{n}(K)$ and $a \in E$.) By construction,

$$
T_{S}:\left(M_{n}(K), \mathcal{D}_{Q}\right) \rightarrow\left(M_{n}(E)^{\rho(G)}, \mathcal{D}_{0}\right)
$$

is an isomorphism. That $\left(M_{n}(K), \mathcal{D}_{Q}\right)$ is trivialized by extending scalars to $E$ follows either from Proposition 2 or directly from the observation that $M_{n}(E)^{\rho(G)} E=$ $M_{n}(E)$. Finally, the associated cocycle is produced from $S$ by the equations $\sigma(S)={ }_{\sigma} D S$. Also, we have $\sigma(F)=d_{\sigma} F C(\sigma)$. Since $S=F^{-1}$, we conclude that $C(\sigma)=d_{\sigma}^{-1}{ }_{\sigma} D$, and hence that $C(\sigma)$ and ${ }_{\sigma} D^{-1}$ produce the same inner automorphism, and hence that the associated cocycle is $\Lambda$.

\section{ExAmple: $K=C$}

As we noted in the introduction, there can be non-trivial DCSAs even in the case that $K$ is algebraically closed. We consider now the case where $K=C$ is algebraically closed and has trivial derivation. In this case, by Proposition 2 , $\left(M_{n}(C), \mathcal{D}_{P}\right)$ is isomorphic to $\left(M_{n}(C), \mathcal{D}_{Q}\right)$ if and only if there is $H \in \mathrm{GL}_{n}(C)$ such that $H^{-1} Q H=P$. If we let $\mathrm{GL}_{n}(C)$ act on $M_{n}(C)$ by conjugation, then 
isomorphism classes of differential matrix algebras correspond to $\mathrm{GL}_{n}(C)$ orbits. These orbits in turn correspond to $C$ matrices in Jordan canonical form, up to the usual permutation of their Jordan blocks.

Note that, in particular, these orbit spaces are infinite. Moreover, we claim that we have an injection

$$
M_{n}(C) / \mathrm{GL}_{n}(C) \rightarrow \mathcal{B} r^{\operatorname{diff}}(K)
$$

This follows from stabilization: there is a map $M_{n}(C) \times M_{m}(C) \rightarrow M_{n m}(C)$ induced from $(P, Q) \mapsto P \otimes I_{m}+I_{n} \otimes Q$ corresponding to the tensor product of differential matrix algebras. These maps are compatible with the $\mathrm{GL}_{n}(C)$ conjugation action. A tensor product with the trivial algebra corresponds to combining with the zero matrix, or $(P, 0) \mapsto P \otimes I_{m}+0 \otimes I_{m}$, which amounts to $P \mapsto P \otimes I_{m}$. As usual, we call this map $M_{n}(C) \rightarrow M_{n m}(C)$ stabilization. If $P_{1}, P_{2} \in M_{n}(C)$ determine the same element of the differential Brauer group, then they become equal after some stabilization, so that $P_{1} \otimes I_{m}$ and $P_{2} \otimes I_{m}$ are conjugate. We can order bases so that matricially $P_{i} \otimes I_{m}$ is in block diagonal form with $m$ diagonal blocks each equal to $P_{i}$. If each $P_{i}$ is in Jordan form, so is $P_{i} \otimes I_{m}$, and it follows that if the latter are conjugate, $P_{1}$ and $P_{2}$ have the same Jordan blocks with the same multiplicities, and hence determine the same element of $M_{n}(C) / \mathrm{GL}_{n}(C)$. Hence the injection to the differential Brauer group.

\section{SplitTing DCSAs}

Let $(A, \mathcal{D})$ be a DCSA over $K$. There is a finite Galois extension $K_{1} \supseteq K$ such that $A \otimes_{K} K_{1}$ is a matrix algebra [6. Cor. 7.8, p. 47]. Also, $K_{1}$ carries a unique derivation making it a PVE of $K$, so that $(A, \mathcal{D}) \otimes_{K} K_{1}$ is a differential matrix algebra over $K_{1}$. By Proposition 2 , there is a PVE $E_{1} \supseteq E$ such that this differential matrix algebra is trivial over $E_{1}$. We want to observe now that $E_{1}$ can be embedded in a PVE $E \supseteq K$, so that $(A, \mathcal{D}) \otimes_{K} E$ is a trivial matrix differential algebra.

The algebra $(A, \mathcal{D})$ plays no role in the construction of $E$ : we simply need to know that a PVE of a finite PVE can be embedded in a PVE. This is obvious if both are finite (and false if neither is).

Proposition 5. A PVE of a finite PVE can be embedded in a PVE.

Proof. Let $E_{1} \supseteq K_{1}$ and $K_{1} \supseteq K$ be PVEs, with $K_{1} \supseteq K$ finite. We suppose that $E_{1} \supseteq K_{1}$ is Picard-Vessiot for the linear operator $L_{1}=X^{(m)}+a_{n-1} X^{(m-1)}+$ $\cdots+a_{0} X^{(0)}$ with $a_{i} \in K_{1}$. For each $\sigma \in G\left(K_{1} / K\right)$, we let $L_{\sigma}$ denote the operator obtained from $L$ by applying $\sigma$ to the coefficients of $L$. Let $K_{2}$ denote a PicardVessiot closure of a Picard-Vessiot closure of $K$ [4, Notation 2, p. 162]. Inside $K_{2}$, we may consider a PVE $E_{\sigma}$ of $K_{1}$ for $L_{\sigma}$ (when $\sigma$ is the identity, $E_{\sigma}=E_{1}$ ), and we let $M$ be a compositum of $E_{\sigma}, \sigma \in G\left(K_{1} / K\right)$. Then $M$ is a PVE of $K_{1}$. It is easy to see that this is a normal extension and that the fixed field is $K$ (use [4, Lemma 20, p. 163] and [3, p. 17]). It is also clear that $M$ is generated over $K$ by a finite dimensional $C$ vector space stable under all the $K$ differential automorphisms, so [2, Prop. 3.9, p. 27] implies that $M$ is a PVE of $K$.

As a corollary, we deduce the existence of Picard-Vessiot splitting fields:

Corollary 1. Let $(A, \mathcal{D})$ be a DCSA over $K$. Then there is a PVE $E$ of $K$ such that $(A, \mathcal{D}) \otimes_{K} E$ is a trivial matrix differential algebra over $E$. 


\section{REFERENCES}

[1] Bass, H., Lectures on topics in algebraic K-theory, Notes by Amit Roy, Tata Institute of Fundamental Research Lectures on Mathematics, 41, Tata Institute of Fundamental Research, Bombay, 1967. MR0279159 (43:4885)

[2] Magid, A., Lectures on Differential Galois Theory, University Lecture Series 7, American Mathematical Society, Providence RI, 1997 (second printing with corrections). MR1301076 (95j:12008)

[3] Magid, A., The Picard-Vessiot antiderivative closure, J. Algebra 244 (2001), 1-18. MR.1856528 (2003a:12005)

[4] Magid, A., The Picard-Vessiot Closure in Differential Galois Theory, in Banach Center Publications 58, Polish Academy of Sciences, Warsaw, 2002, 151-164. MR 1972451|(2004d:12010)

[5] Roy, A., and Sridharan, R., Derivations in Azumaya algebras, J. Math. Kyoto Univ. 7 (1967), 161-167. MR0222073 (36:5125)

[6] Saltman, D., Lectures on division algebras, CBMS Regional Conference Series in Mathematics, 94, American Mathematical Society, Providence, RI, on behalf of the Conference Board of the Mathematical Sciences, Washington, DC, 1999. MR1692654 (2000f:16023)

[7] van der Put, M., and Singer, M., Galois Theory of Linear Differential Equations, SpringerVerlag, New York, 2003. MR1960772 (2004c:12010)

Department of Mathematics, Texas Tech University, Lubbock, Texas 79409

Department of Mathematics, University of Oklahoma, Norman, Oklahoma 73019 